\title{
EFEITO DA TEMPERATURA NO ARMAZENAMENTO DE UVAS APIRÊNICAS MINIMAMENTE PROCESSADAS ${ }^{1}$
}

\author{
BEN-HUR MATTIUZ ${ }^{2}$, ANA CAROLINAALMEIDA MIGUEL ${ }^{3}$, \\ VANESSA CURY GALATI ${ }^{4}$, JAIR COSTA NACHTIGAL $^{5}$
}

RESUMO - O trabalho teve como objetivo avaliar a qualidade pós-colheita de três cultivares de uvas de mesa sem semente submetidas ao processamento mínimo e armazenadas sob refrigeração e à temperatura ambiente. Para tanto, foram utilizadas uvas das cultivares BRS Clara, BRS Linda e BRS Morena, produzidas na Embrapa Uva e Vinho/Estação Experimental de Viticultura Tropical, em Jales-SP. Os cachos, depois de higienizados e imersos em água clorada a $200 \mathrm{mg}$ de cloro. $\mathrm{L}^{-1}$ por 5 minutos, foram mantidos em câmara fria, a $12^{\circ} \mathrm{C}$, por $12 \mathrm{~h}$. As bagas foram degranadas e lavadas em solução de álcool a 70\%, por 5 segundos. Depois de escorrido o excesso da solução alcoólica, as bagas foram acondicionadas em bandejas de tereftalato de polietileno (PET) transparente com tampa e com capacidade para $500 \mathrm{~mL}$. Cada unidade, contendo $200 \mathrm{~g}$ de bagas, foi armazenada a $12 \pm 1,8^{\circ} \mathrm{C}$ e $24 \pm 0,8^{\circ} \mathrm{C}$, por 12 dias. Avaliaram-se, a cada três dias, a perda de massa fresca, a aparência, a coloração e os teores de sólidos solúveis (SS) e de acidez titulável (AT). A temperatura de $12^{\circ} \mathrm{C}$ manteve a turgidez, a coloração, as qualidades organoléptica (relação SS/AT) e comercial das bagas das três cultivares testadas, por nove dias, enquanto no armazenamento à temperatura ambiente $\left(24^{\circ} \mathrm{C}\right)$, ocorre perda da qualidade comercial das bagas aos três dias para as cvs. BRS Clara e BRS Linda, e aos seis dias para a cv. BRS Morena. Termos para indexação: Vitis sp., uvas sem sementes, pós-colheita, processamento mínimo.

\section{EFFECT OF STORED TEMPERATURE IN MINIMALLY PROCESSED SEEDLESS TABLE GRAPES}

\begin{abstract}
The objective of this research was to evaluate the postharvest quality of three apirenic (seedless) table grapes cultivars submitted to minimally processed and stored under refrigeration and room temperature. It was used the cultivars BRS Clara, BRS Linda and BRS Morena produced at Embrapa Grapes and Wine/Tropical Viticulture of Experimental Station, in Jales, state of São Paulo. The clusters were immersed in a solution of sodium hypochlorite $\left(200 \mathrm{mg} \mathrm{Cl} . \mathrm{L}^{-1}\right)$ for 5 minutes before being stored at $12^{\circ} \mathrm{C}$ for 12 hours. The berries were removed and rinsed with alcohol solution (70\%) per 5 seconds. After drained the water excess, the berries were conditioned in $500 \mathrm{~mL}$ capacity trays of transparent tereftalato of polyethylene (PET) with lid. Each unit with $200 \mathrm{~g}$ of berries was stored at $12 \pm 1.8^{\circ} \mathrm{C}$ and $24 \pm 0.8^{\circ} \mathrm{C}$ during 12 days. Every three days, the loss of weight, appearance, color, soluble solids (SS) and titratable acidity (TA) were evaluated. The temperature of $12^{\circ} \mathrm{C}$ kept the turgidity, the color, the ratio SS:TA and the marketing conditions of the berries from the three cultivars tested after nine days of evaluations. The berries stored at room temperature $\left(24^{\circ} \mathrm{C}\right)$, loss the commercial quality at the third day for BRS Clara and BRS Linda cultivars and at the sixth day for the BRS Morena cultivar.
\end{abstract}

Index terms: Vitis sp., postharvest, minimally processed.

\footnotetext{
${ }^{1}$ (Trabalho 104-08). Recebido em: 30-08-2008. Aceito para publicação em: 13-01-2009.

${ }^{2}$ Eng. Agr., Dr., Professor do Departamento de Tecnologia da Universidade Estadual Paulista. UNESP-FCAV, Campus de Jaboticabal. Via de Acesso Prof. Paulo Donato Castellane, s/n. CEP: 14.884-900. Jaboticabal-SP. E-mail: benhur@fcav.unesp.br ${ }^{3} \mathrm{Eng}^{\mathrm{a}} \mathrm{Agr}^{\mathrm{a}}$ formada pela Universidade Estadual Paulista, UNESP-FCAV. E-mail: acmiguel@esalq.usp.br; vanessagalati@ig.com.br

${ }^{4}$ Eng. Agr., Dr., Pesquisador da Embrapa Clima Temperado. Rodovia BR 392, km 78. CP 403 CEP: 96001-970. Pelotas-RS. E-mail: jair@cpact.embrapa.br
} 


\section{INTRODUÇÃO}

A produção mundial de uvas de mesa foi de 1,17 milhãos de toneladas em 2005, sendo que a China e a Turquia figuram como os maiores produtores (Agrianual, 2006).

Nesta última década, as variedades de uvas sem sementes têm despertado crescente interesse dos produtores, dada a tendência de consumo do mercado internacional e a presença cada vez mais forte desse tipo de produto nos supermercados brasileiros (Leão \& Pereira, 2001).

Em 2003, a Embrapa Uva e Vinho lançou as primeiras cultivares de uva de mesa sem semente BRS Clara, BRS Linda e BRS Morena - com alta fertilidade natural nas condições tropicais do Brasil e com frutas de boa qualidade. A cv. BRS Morena foi obtida do cruzamento 'Marroo Seedless' x 'Centennial Seedless', realizado em 1998, e apresenta produtividade de 20 a 25 t.ha $^{-1}$, bagas de coloração preta, textura crocante e elevado grau glucométrico. A 'BRS Clara', obtida do cruzamento CNPUV 154$147 \mathrm{X}$ 'Centennial Seedless', é uma cultivar produtiva (cerca de $30 \mathrm{t} \mathrm{ha}^{-1}$. ano ${ }^{-1}$ ), que apresenta cacho de tamanho médio a grande; baga elíptica, verdeamarelada, chegando a amarela quando exposta ao sol; polpa incolor e firme; sabor moscatel leve e agradável, e traço de semente grande, porém imperceptível à mastigação. A cv. BRS Linda foi obtida do cruzamento CNPUV 154-90 x 'Saturn', ambas uvas sem sementes, realizado em 1998, que apresenta coloração verde, tonalidade preferida em certos mercados, como o inglês; limitado potencial glucométrico, normalmente na faixa de 14 a $15^{\circ}$ Brix, polpa firme e crocante. $\mathrm{O}$ sabor neutro faz dela uma uva bem aceita pelo consumidor brasileiro que, normalmente, prefere frutas menos ácidas (Camargo et al., 2003).

Conforme Lima et al. (2000), os principais problemas de conservação pós-colheita das uvas de mesa são a ocorrência de danos mecânicos, podridões, escurecimento das bagas, secamento do engaço e degrana, o que compromete a aceitação pelos consumidores, muitos dos quais estão atentos aos benefícios de uma vida saudável, que incluem o consumo de uma variedade de frutas na dieta diária. Atualmente, os consumidores buscam alimentos que se encontrem prontos para o consumo, dispensando as operações de descascamento e corte (Allong et al., 2000).

De acordo com Cantwell (1992) e Wiley (1997), o processamento mínimo tem por objetivo fornecer ao consumidor um produto fresco, conveniente, com qualidade e garantia de sanidade. Esses produtos, além de consumidos em menor tempo, proporcionam agregação de valor ao produto agrícola, redução das perdas pós-colheita e meios alternativos de comercialização (Chitarra, 1998). Em se tratando de uma fruta perecível, o processamento mínimo poderia ser uma alternativa interessante para a uva, pois permitiria valorizar as bagas com boa qualidade, provenientes de cachos que não atinjam os padrões para comercialização na forma de fruta fresca.

Entretanto, o conhecimento sobre os aspectos fisiológicos, químicos e bioquímicos de produtos minimamente processados ainda é pequeno, e as operações envolvidas no processamento mínimo de frutas reduzem a vida útil das mesmas (Cantwell, 1992; Durigan \& Sargent, 1999). Para controlar ou minimizar essas consequências negativas, a temperatura de armazenamento é um fator importante, pelo fato de reduzir o metabolismo vegetal, retardar o crescimento microbiano e reduzir o aparecimento de deteriorações (Brecht, 1995; Cantwell, 2000).

O objetivo deste trabalho foi avaliar a qualidade pós-colheita de três cultivares de uvas de mesa sem sementes submetidas ao processamento mínimo e armazenadas sob refrigeração e na temperatura ambiente.

\section{MATERIAL E MÉTODOS}

Foram utilizadas uvas de mesa apirênicas (sem sementes) das cultivares BRS Morena, BRS Linda e BRS Clara, procedentes da Estação Experimental de Viticultura Tropical, Jales-SP, pertencente à Embrapa Uva e Vinho.

As uvas foram colhidas 120 dias após a poda e acondicionadas em caixas de papelão com tampa. Os cachos foram colhidos pela manhã e transportados em condição climatizada, de forma rápida e cuidadosa, ao Laboratório de Tecnologia dos Produtos Agrícolas da Universidade Estadual Paulista, Faculdade de Ciências Agrárias e Veterinárias, Câmpus de Jaboticabal, São Paulo.

No laboratório, os cachos foram higienizados por imersão em água clorada a $200 \mathrm{mg}$ de cloro.L-1 por 5 minutos, e, após, mantidos em câmara fria, a $12^{\circ} \mathrm{C}$, por $12 \mathrm{~h}$. Pessoas treinadas e com proteção individual adequada (luvas, máscaras, protetores de cabelo e macacões) procederam à degrana das bagas. Em seguida, procedeu-se à imersão das bagas em solução de álcool a 70\%, por cinco segundos, para desinfecção superficial (Litcher et al., 2002). A degrana foi realizada manualmente, cortando-se o pedicelo junto à baga . Em seguida, estas foram 
colocadas em peneiras plásticas para escorrer o excesso de água e, logo após, acondicionadas em bandejas de tereftalato de polietileno (PET) transparente com tampa e com capacidade para 500 $\mathrm{mL}$ (Neoform ${ }^{\circledR}$ N-90). As unidades, contendo $200 \mathrm{~g}$ do produto, foram armazenadas sob refrigeração (12 $\left.\pm 1,8^{\circ} \mathrm{C}\right)$ e em temperatura ambiente $\left(24 \pm 0,8^{\circ} \mathrm{C}\right)$.

A cada três dias, foram avaliadas a perda de massa fresca, mediante a pesagem em balança analítica, o que permitiu expressar as variações médias acumuladas em porcentagem, e a aparência, determinada por meio da atribuição de notas, sendo: 4 (ótima) $=$ baga túrgida, sem fungos e com a coloração característica da cultivar; 3 (boa) = baga sem brilho, túrgida, sem fungos e com a coloração característica da cultivar; 2 (aceitável) = baga sem brilho, com perda da turgidez e sem fungos, porém escurecida; 1 (ruim) = baga murcha, com fungos e escurecida. Atribuiu-se a nota 2 como sendo condição para descarte comercial. Na mesma ocasião, foram realizadas análises de coloração utilizando-se de reflectômetro Minolta CR-200b, que permitiu determinar a luminosidade $(L)$, o ângulo de cor $(H u e)$ e a cromaticidade $(C)$ das bagas (Minolta Corp., 1994). Os teores de sólidos solúveis (SS) e de acidez titulável (AT) foram determinados conforme método no 932-12 e 942,15 da AOAC (1987), respectivamente.

O experimento foi conduzido em delineamento inteiramente casualizado, em esquema fatorial composto por dois fatores: temperatura (12 e $\left.24^{\circ} \mathrm{C}\right)$ e dias de armazenamento $(0 ; 3 ; 6 ; 9$ e 12$)$. Foram utilizadas duas bandejas (repetições) para as determinações químicas, das quais se tomavam 20 bagas para a determinação da coloração.

Os dados obtidos foram submetidos à análise de variância, pelo teste F, e as médias, comparadas mediante o teste de Tukey, em nível de $5 \%$ de probabilidade (SAS Institute Inc., 2004).

\section{RESULTADOS E DISCUSSÃO}

Analisando-se o efeito da temperatura na perda de massa (Figura 1), percebe-se que as bagas armazenadas à temperatura ambiente foram as que apresentaram as maiores perdas durante o armazenamento, sendo que a cv. BRS Morena apresentou maior valor acumulado (2,0\%), aos 12 dias de armazenamento, seguido das cvs. BRS Clara e BRS Linda. Esses resultados eram esperados, uma vez que as baixas temperaturas, associadas à proteção oferecida pelas embalagens, retardam a perda de água das bagas, além de reduzirem o metabolismo das frutas pela diminuição de sua taxa respiratória, da redução de sua atividade enzimática e da perda de vapor de água, refletindo diretamente na perda de peso (Chitarra \& Chitarra, 2005). Os valores de perda de massa obtidos, aos 12 dias para as três cultivares apirênicas mantidas a $12^{\circ} \mathrm{C}$, foram inferiores aos citados por Kluge et al. (2002), que consideram que as uvas podem perder até $1,2 \%$ de água, sem que haja prejuízo à aparência e comprometimento das características organolépticas. Porém, perdas acima de 5 a $6 \%$ são suficientes para causar o murchamento das bagas (Chitarra \& Chitarra, 2005).

Alguns parâmetros relacionados à aparência das bagas foram afetados nas duas temperaturas de armazenamento, conforme o exposto na Figura 2. As três cultivares apresentaram melhor manutenção da qualidade comercial quando armazenadas a $12^{\circ} \mathrm{C}$, atingindo a nota para o descarte comercial (nota 2) aos 9 dias de armazenamento. Na temperatura ambiente $\left(24^{\circ} \mathrm{C}\right)$, todas as cultivares mostraram rápida deterioração. Mattiuz et al. (2004) não observaram perda da qualidade comercial de uvas 'BRS Morena' minimamente processada até o $12^{\circ}$ dia de armazenamento, a $2,5^{\circ} \mathrm{C}$. Provavelmente, a baixa temperatura empregada promoveu maior redução do metabolismo e consequente melhora na manutenção da qualidade dos produtos processados, quando comparados com este experimento $\left(12\right.$ e $\left.24^{\circ} \mathrm{C}\right)$.

Frutas minimamente processadas apresentam vida útil muito curta em comparação às frutas intactas (Cantwell, 1992), pois as operações envolvidas no preparo do produto reduzem a vida de prateleira das mesmas. Sarzi (2002) obteve viabilidade comercial de 6 e 7 dias para abacaxi 'Pérola' e mamão 'Formosa', respectivamente, minimamente processados e armazenados a $9^{\circ} \mathrm{C}$. Pinto (2002) verificou manutenção da aparência satisfatória por 12 dias, em melões 'Orange Fresh', minimamente processados e armazenados a $6^{\circ} \mathrm{C}$. A aparência é o fator de qualidade mais importante na determinação do valor de comercialização do produto, podendo ser avaliada pelo frescor, cor, forma, higiene e ausência de defeitos (Chitarra \& Alves, 2001; Chitarra \& Chitarra, 2005).

Quanto à coloração, verificou-se que, para a cv. BRS Clara, a temperatura não influenciou no escurecimento, representado pelo fator L, porém o período de armazenamento proporcionou um decréscimo significativo da luminosidade das bagas a partir do $6^{\circ}$ dia, indicando escurecimento generalizado das bagas (Tabela 1). As modificações observadas no ângulo de cor e cromaticidade, durante o período de armazenamento, indicam que as bagas das cvs. BRS Clara e BRS Linda, armazenadas nas duas temperaturas (Tabelas 1 e 2 , respectivamente), 
apresentaram amarelecimento, característico de senescência. Entretanto, na cv. BRS Morena, por ser uma cultivar de película escura, houve aumento significativo dos valores do ângulo Hue e da luminosidade na temperatura de $24^{\circ} \mathrm{C}$ e aumento dos valores da cromaticidade durante o armazenamento. Associado à coloração, o brilho superficial é um atributo de qualidade bastante relevante (Chitarra \& Alves, 2001). No caso das uvas, a coloração das bagas é o fator mais importante no processo de escolha pelos consumidores, sendo responsável pela qualidade comercial dessas frutas.

Em relação à cv. BRS Linda, a análise da variância revelou que as bagas armazenadas à temperatura ambiente apresentaram menor valor médio de luminosidade que as mantidas a $12^{\circ} \mathrm{C}$, apresentando aspecto escurecido (Tabela 2). Neste caso, provavelmente, a temperatura mais elevada acelerou as reações enzimáticas ligadas ao escurecimento, características da senescência do produto. Observa-se, ainda, o efeito positivo da temperatura de $12^{\circ} \mathrm{C}$ na manutenção da qualidade inicial, evidenciada pelo maior ângulo de cor (Tabela 2). Comportamento similar foi observado na cv. BRS Morena, para a qual o armazenamento com menor temperatura propiciou a obtenção de bagas com coloração mais próxima do azul (ângulo de cor menor) (Tabela 3).

Pela semelhança nos valores de luminosidade e ângulo de cor ao longo do armazenamento, podese observar que as cultivares não apresentaram mudança apreciável na coloração durante o período de avaliação. Segundo Chitarra (1999), a mudança de coloração tem sido um dos maiores problemas na conservação dos produtos minimamente processados, sendo considerado um fator- chave na aceitação comercial destes. Esses resultados concordam com o enunciado por Wiley (1997) que o uso de temperaturas de armazenamento mais baixas reduz as alterações na coloração dos produtos minimamente processados. Essas alterações podem ser resultado da oxidação de fenóis que, estimulados pelas injúrias devidas às operações envolvidas no processamento mínimo, provocam alterações na cor de bagas de uvas armazenadas (Lima et al., 2000). A intensidade da coloração final dos produtos minimamente processados depende da cultivar e da quantidade e qualidade dos pigmentos que neles se encontram. Pinto (2002) também observou a manutenção da coloração durante os três dias em que foram armazenadas melancias minimamente processadas.

Pode-se verificar, na Tabela 4, que houve pouca variação nos valores médios para os teores de sólidos solúveis (SS) para as três cultivares, nas duas temperaturas de armazenamento, porém as uvas mantidas à temperatura ambiente apresentaram os menores teores de açúcares e os maiores de acidez titulável (AT), o que resultou em uma relação SS/AT inferior àquelas armazenadas a $12^{\circ} \mathrm{C}$. Neste trabalho, a relação SS/AT foi influenciada pela temperatura, pelo período de armazenamento e pela interação entre esses fatores. O desequilíbrio entre os teores de açúcares e ácidos ao longo do período de armazenamento, nas uvas armazenadas à temperatura ambiente, permite inferir que estas apresentaram perda da qualidade organoléptica.

De acordo com os resultados encontrados, pode-se verificar que as cultivares testadas, com exceção da 'BRS Linda', atendem às normas internacionais de comercialização para as uvas de mesa, a qual exige o teor mínimo de SS de $14^{\circ}$ Brix (Kader, 1992; Barros et al., 1995). De acordo com Coombe (1992), o decréscimo no teor de sólidos solúveis está associado à utilização dos açúcares como substratos na atividade respiratória. Detoni et al. (2005) observaram manutenção da doçura em uvas 'Niágara Rosada' armazenadas a $14^{\circ} \mathrm{C}$, por 28 dias. Contrariamente, os mesmos autores constataram perdas na qualidade e na doçura, durante o armazenamento de uvas a $24^{\circ} \mathrm{C}$, por 21 dias.

Pela análise da acidez titulável, verifica-se que, no armazenamento a $12^{\circ} \mathrm{C}$, as cultivares não apresentaram variações significativas ao longo do armazenamento (Tabela 4). Entretanto, para a temperatura de $24^{\circ} \mathrm{C}$, houve acréscimo neste teor ao longo do armazenamento, devido, provavelmente, à reconversão dos açúcares em ácidos, observado pela diminuição dos SS, como suprimento para o processo respiratório, via ciclo de Krebs, culminando com seu acúmulo. Segundo Al Kaisy et al. (1981) e Ruffner et al. (1983), os ácidos orgânicos estão entre os principais substratos utilizados na atividade respiratória da uva. Grangeiro et al. (2002), ao avaliarem a conservação de uvas 'Superior Seedless' submetidas ao armazenamento refrigerado, verificaram que o teor de acidez titulável se manteve inalterado durante o armazenamento. A manutenção dos teores da acidez titulável na menor temperatura é um indicativo de que o metabolismo permaneceu quase inalterado para as cultivares citadas, ou com pequenas transformações, sem causar grandes variações nos resultados. Isso possibilitou melhor manutenção da qualidade organoléptica dos produtos, indicada pela relação SS/AT, ao longo do armazenamento. A relação entre os teores de SS e AT, conhecida como índice de maturação, é um importante parâmetro qualitativo, pois indica o sabor 
do produto, uma vez que é o resultado do balanço entre os constituintes ácidos e doces da fruta. Para o mercado interno de frutas, uma relação SS/AT elevada é desejável (Thé et al., 2001).

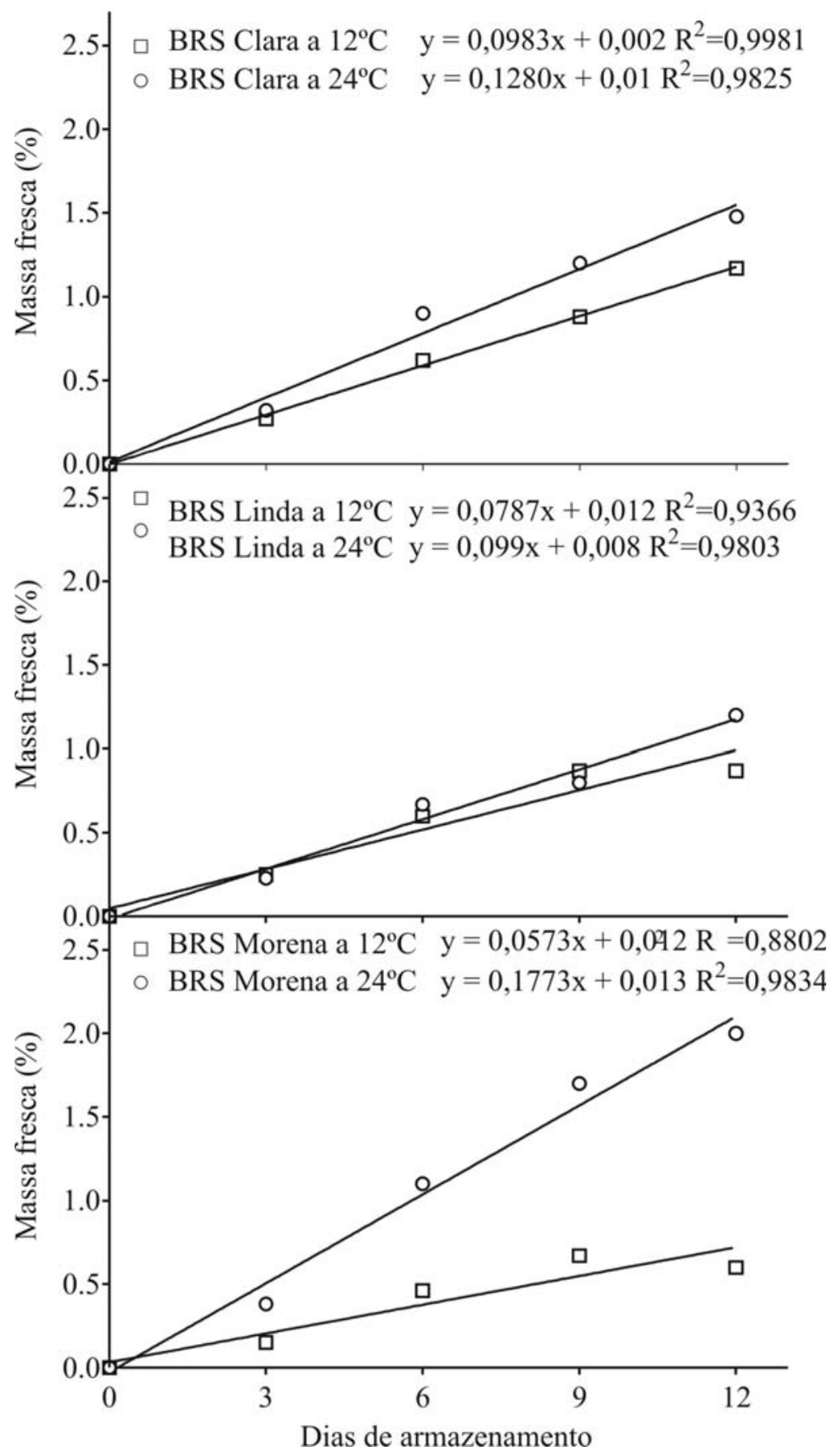

FIGURA 1 - Perda acumulada de massa fresca, em porcentagem, de três cultivares de uvas de mesa apirênicas minimamente processadas e armazenadas sob duas temperaturas. 


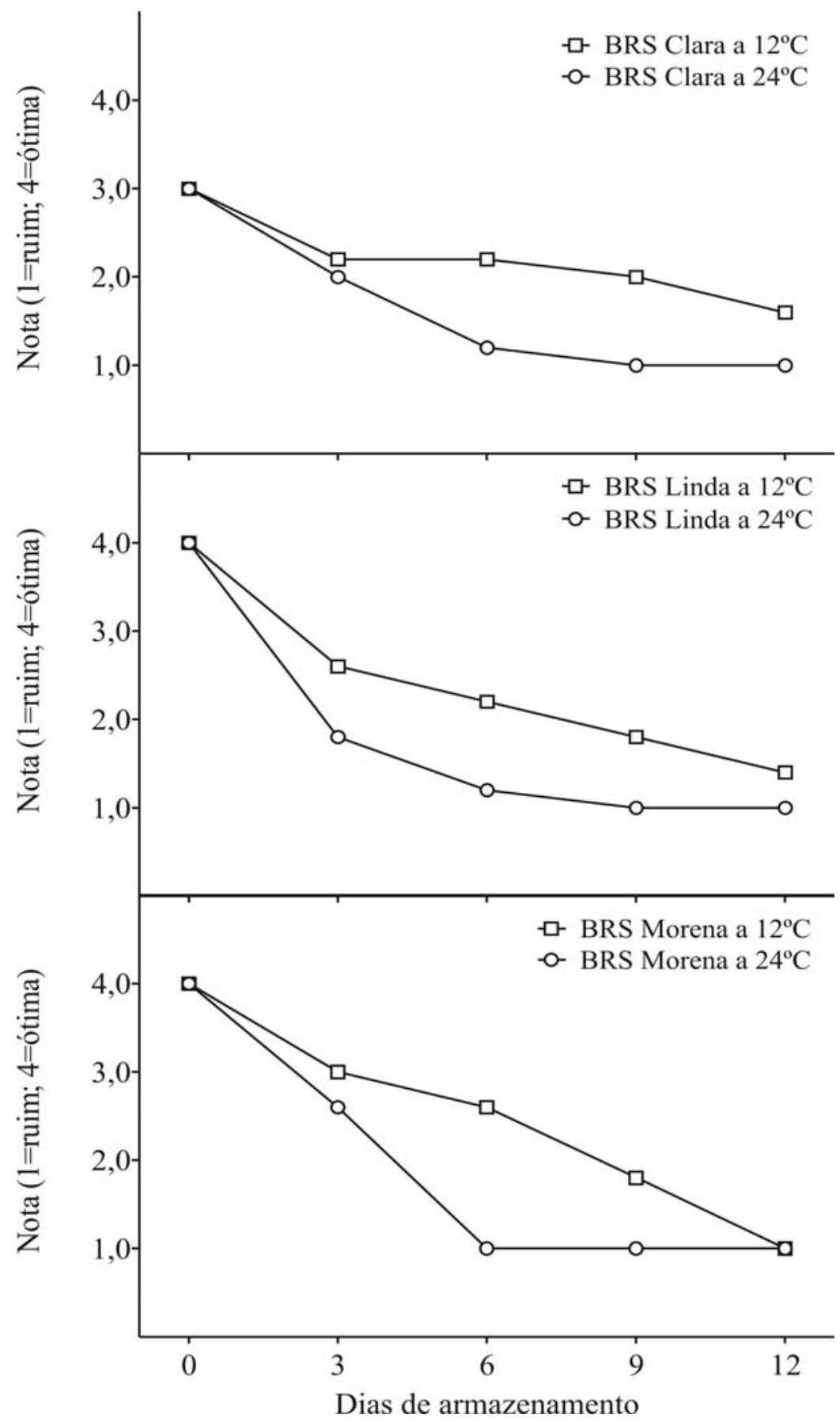

FIGURA 2-Evolução da aparência de três cultivares de uvas de mesa apirênicas minimamente processadas e armazenadas sob duas temperaturas. 
TABELA 1 - Evolução da coloração externa de uvas de mesa apirênicas cv. BRS Clara submetidas ao processamento mínimo e armazenadas sob duas temperaturas.

\begin{tabular}{cccc}
\hline \multicolumn{1}{c}{ Fator } & $\boldsymbol{L}$ & Hue & $\boldsymbol{C}$ \\
\hline $\begin{array}{c}\text { Temperatura } \\
12^{\circ} \mathrm{C}\end{array}$ & $41,82 \mathrm{~A}$ & $116,10 \mathrm{~A}$ & $12,85 \mathrm{~A}$ \\
$24^{\circ} \mathrm{C}$ & $41,74 \mathrm{~A}$ & $111,24 \mathrm{~B}$ & $11,80 \mathrm{~B}$ \\
& & & \\
Dias & & & \\
0 & $43,11 \mathrm{~A}$ & $116,70 \mathrm{~A}$ & $12,97 \mathrm{~A}$ \\
3 & $43,03 \mathrm{~A}$ & $116,23 \mathrm{~A}$ & $12,93 \mathrm{~A}$ \\
6 & $41,39 \mathrm{~B}$ & $116,84 \mathrm{~A}$ & $11,63 \mathrm{~A}$ \\
9 & $41,29 \mathrm{~B}$ & $111,66 \mathrm{~B}$ & $12,29 \mathrm{~A}$ \\
12 & $40,08 \mathrm{~B}$ & $106,91 \mathrm{C}$ & $11,81 \mathrm{~A}$ \\
\hline C.V. $(\%)$ & 3,61 & 5,20 & 15,89 \\
\hline
\end{tabular}

$L=$ luminosidade; $H u e=$ ângulo de cor; $C=$ cromaticidade. Médias para cada fator, seguidas de pelo menos uma letra comum, nas colunas, não diferem significativamente entre si, pelo teste de Tukey $(\mathrm{P}<0,05)$.

TABELA 2 - Evolução da coloração externa de uvas de mesa apirênicas cv. BRS Linda submetidas ao processamento mínimo e armazenadas sob duas temperaturas.

\begin{tabular}{cccc}
\hline \multicolumn{1}{c}{ Fator } & \multicolumn{1}{c}{$\boldsymbol{H}$} & $\boldsymbol{C} \boldsymbol{e}$ & \multicolumn{1}{c}{$\boldsymbol{C}$} \\
\hline Temperatura & & & \\
$12^{\circ} \mathrm{C}$ & $40,90 \mathrm{~A}$ & $127,62 \mathrm{~A}$ & $8,77 \mathrm{~B}$ \\
$24^{\circ} \mathrm{C}$ & $39,31 \mathrm{~B}$ & $121,71 \mathrm{~B}$ & $9,14 \mathrm{~A}$ \\
& & & \\
Dias & & & \\
0 & $40,39 \mathrm{~A}$ & $129,40 \mathrm{~A}$ & $9,19 \mathrm{AB}$ \\
3 & $39,91 \mathrm{~A}$ & $127,93 \mathrm{~A}$ & $9,03 \mathrm{~B}$ \\
6 & $39,65 \mathrm{~B}$ & $125,56 \mathrm{~A}$ & $8,62 \mathrm{~B}$ \\
9 & $39,80 \mathrm{AB}$ & $125,98 \mathrm{~A}$ & $9,36 \mathrm{AB}$ \\
12 & $40,77 \mathrm{~A}$ & $114,45 \mathrm{~B}$ & $10,52 \mathrm{~A}$ \\
\hline C.V. $(\%)$ & 2,88 & 6,58 & 17,94 \\
\hline
\end{tabular}

$L=$ luminosidade Hue = ângulo de cor; $C=$ cromaticidade. Médias para cada fator, seguidas de pelo menos uma letra comum, nas colunas, não diferem significativamente entre si, pelo teste de Tukey $(\mathrm{P}<0,05)$.

TABELA 3 - Evolução da coloração externa de uvas de mesa apirênicas cv. BRS Morena submetidas ao processamento mínimo e armazenadas sob duas temperaturas.

\begin{tabular}{cccl}
\hline \multicolumn{1}{c}{ Fator } & $\boldsymbol{L}$ & $\boldsymbol{H}$ Hue & \multicolumn{1}{c}{$\boldsymbol{C}$} \\
\hline Temperatura & & & \\
$12^{\circ} \mathrm{C}$ & $27,66 \mathrm{~B}$ & $331,99 \mathrm{~B}$ & $3,61 \mathrm{~A}$ \\
$24^{\circ} \mathrm{C}$ & $28,73 \mathrm{~A}$ & $340,12 \mathrm{~A}$ & $4,01 \mathrm{~A}$ \\
& & & \\
Dias & & & \\
0 & $28,68 \mathrm{~A}$ & $337,10 \mathrm{~A}$ & $3,85 \mathrm{AB}$ \\
3 & $27,89 \mathrm{~A}$ & $341,42 \mathrm{~A}$ & $2,87 \mathrm{~B}$ \\
6 & $28,40 \mathrm{~A}$ & $329,37 \mathrm{~A}$ & $3,68 \mathrm{AB}$ \\
9 & $28,11 \mathrm{~A}$ & $335,52 \mathrm{~A}$ & $4,12 \mathrm{~A}$ \\
12 & $27,90 \mathrm{~A}$ & $336,89 \mathrm{~A}$ & $4,53 \mathrm{~A}$ \\
\hline C.V. $(\%)$ & 3,77 & 6,08 & 32,22 \\
\hline
\end{tabular}

$L=$ luminosidade; Hue = ângulo de cor; $C=$ cromaticidade. Médias para cada fator, seguidas de pelo menos uma letra comum, nas colunas, não diferem significativamente entre si, pelo teste de Tukey $(\mathrm{P}<0,05)$. 
TABELA 4 - Interação dos fatores de dias de armazenamento e temperatura para os teores de sólidos solúveis (SS), acidez titulável (AT), relação SS/AT de três cultivares de uvas de mesa apirênicas submetidas ao processamento mínimo.

\begin{tabular}{|c|c|c|c|c|c|c|}
\hline \multirow{2}{*}{ Dias } & \multicolumn{2}{|c|}{ SS $\left({ }^{\circ}\right.$ Brix $)$} & \multicolumn{2}{|c|}{ AT $(\%)$} & \multicolumn{2}{|c|}{ SS/AT } \\
\hline & $12^{\circ} \mathrm{C}$ & $24^{\circ} \mathrm{C}$ & $12^{\circ} \mathrm{C}$ & $24^{\circ} \mathrm{C}$ & $12^{\circ} \mathrm{C}$ & $24^{\circ} \mathrm{C}$ \\
\hline & \multicolumn{6}{|c|}{ 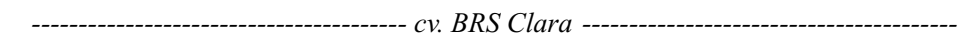 } \\
\hline 0 & $22,22 \mathrm{Aa}$ & $22,22 \mathrm{Aa}$ & $0,94 \mathrm{Aa}$ & $0,94 \mathrm{Ca}$ & $23,73 \mathrm{Ba}$ & $23,73 \mathrm{Ba}$ \\
\hline 3 & $22,57 \mathrm{Aa}$ & $22,30 \mathrm{Aa}$ & $0,86 \mathrm{Aa}$ & $0,81 \mathrm{Ca}$ & $26,29 \mathrm{Aa}$ & $27,50 \mathrm{Aa}$ \\
\hline 6 & $22,54 \mathrm{Aa}$ & $22,38 \mathrm{Aa}$ & $0,78 \mathrm{Ab}$ & $0,98 \mathrm{BCa}$ & $28,93 \mathrm{Aa}$ & $22,95 \mathrm{Bb}$ \\
\hline 9 & $21,79 \mathrm{Aa}$ & $21,21 \mathrm{Ba}$ & $0,82 \mathrm{Ab}$ & $1,23 \mathrm{Ba}$ & $25,56 \mathrm{Ba}$ & $17,30 \mathrm{Cb}$ \\
\hline \multirow[t]{2}{*}{12} & $21,70 \mathrm{Aa}$ & $21,16 \mathrm{Ba}$ & $0,74 \mathrm{Ab}$ & $1,55 \mathrm{Aa}$ & $24,04 \mathrm{Ba}$ & $13,88 \mathrm{Db}$ \\
\hline & \multicolumn{6}{|c|}{---------------------------------------- cv. BRS Linda ---------------------------------------- } \\
\hline 0 & $13,24 \mathrm{Aa}$ & $13,24 \mathrm{Aa}$ & $0,52 \mathrm{Aa}$ & $0,52 \mathrm{Ba}$ & $25,28 \mathrm{Aa}$ & $25,28 \mathrm{Aa}$ \\
\hline 3 & $13,32 \mathrm{Aa}$ & $13,05 \mathrm{ABa}$ & $0,56 \mathrm{Aa}$ & $0,53 \mathrm{Ba}$ & $23,69 \mathrm{Aa}$ & $24,67 \mathrm{Aa}$ \\
\hline 6 & $12,65 \mathrm{Ba}$ & $12,65 \mathrm{BCa}$ & $0,46 \mathrm{Ab}$ & $0,61 \mathrm{Ba}$ & $27,65 \mathrm{Aa}$ & $20,77 \mathrm{Ab}$ \\
\hline 9 & $12,81 \mathrm{Ba}$ & $12,30 \mathrm{Cb}$ & $0,47 \mathrm{Ab}$ & $0,61 \mathrm{Ba}$ & $27,37 \mathrm{Aa}$ & $21,63 \mathrm{Ab}$ \\
\hline \multirow[t]{2}{*}{12} & $12,90 \mathrm{Ba}$ & $11,65 \mathrm{Db}$ & $0,54 \mathrm{Ab}$ & $1,46 \mathrm{Aa}$ & $24,23 \mathrm{Aa}$ & $8,15 \mathrm{Bb}$ \\
\hline & \multicolumn{6}{|c|}{ 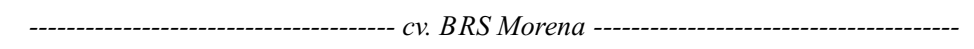 } \\
\hline 0 & $15,65 \mathrm{ABa}$ & $15,65 \mathrm{Aa}$ & $0,64 \mathrm{Aa}$ & $0,64 \mathrm{BCa}$ & $24,52 \mathrm{Aa}$ & $24,52 \mathrm{Aa}$ \\
\hline 3 & $14,95 \mathrm{Ca}$ & $15,22 \mathrm{ABa}$ & $0,59 \mathrm{Aa}$ & $0,58 \mathrm{Ca}$ & $25,33 \mathrm{Aa}$ & $26,21 \mathrm{Aa}$ \\
\hline 6 & $15,92 \mathrm{Aa}$ & $14,73 \mathrm{BCb}$ & $0,58 \mathrm{Ab}$ & $0,88 \mathrm{Aa}$ & $27,63 \mathrm{Aa}$ & $17,23 \mathrm{Bb}$ \\
\hline 9 & $15,11 \mathrm{BCa}$ & $14,65 \mathrm{Cb}$ & $0,59 \mathrm{Ab}$ & $0,72 \mathrm{Ba}$ & $25,45 \mathrm{Aa}$ & $20,56 \mathrm{Bb}$ \\
\hline 12 & $15,16 \mathrm{Ca}$ & $14,76 \mathrm{BCb}$ & $0,56 \mathrm{Ab}$ & $0,86 \mathrm{Aa}$ & $26,94 \mathrm{Aa}$ & $17,24 \mathrm{Bb}$ \\
\hline
\end{tabular}

Médias seguidas de pelo menos uma letra comum, minúscula nas linhas e maiúscula nas colunas, para cada cultivar e para cada variável, não diferem significativamente entre si, pelo teste de Tukey $(\mathrm{P}<0,05)$.

\section{CONCLUSÕES}

1-A temperatura de $12^{\circ} \mathrm{C}$ mantém a turgidez, a coloração, as qualidades organolépticas (relação SS/AT) e comercial das bagas das cultivares BRS Clara, BRS Linda e BRS Morena por nove dias.

2-No armazenamento à temperatura ambiente $\left(24^{\circ} \mathrm{C}\right)$, ocorre perda da qualidade comercial das bagas aos três dias, para as cvs. BRS Clara e BRS Linda, e aos seis dias, para a cv. BRS Morena.

\section{REFERÊNCIAS}

AGRIANUAL 2006: anuário da agricultura brasileira. São Paulo: FNP, Consultórios e Comércio, 2006. p.493504.

AL KAISY, A. M.; SACHDE, A.G.; GHALIB, H.A.;HAMEL, S.M. Physical and chemical changes during ripening of some grape varieties grown in Basrah. Amercian Journal of Enology and Viticulture, Davis, v.32, n.4, p.268-271, 1981.

ALLONG, R.; WICKHAM, L.D.; MOHAMMED, M. The effect of cultivar, fruit ripeness, storage temperature and duration on quality of fresh-cut mango. Acta Horticulture, Wageningen, n.509, p.487494, 2000.

AOAC. Official methods of analysis of the Association of Official Analytical Chemists. $13^{\text {th }} \mathrm{ed}$. Washington: Ed. Patricia Cuniff, 1987. v.2. cap.37.

BARROS, J.C. da S.M. de; FERRI, C.P.; OKAWA, H. Qualidade da uva fina de mesa comercializada na Ceasa de Campinas, 1993-94. Informações Econômicas, São Paulo, v.25, n.7, p.53-61, 1995.

BRECHT, J.K. Physiology of lightly processed fruits and vegetables. HortScience, Alexandria, v.30, n.1, p.18-22, 1995.

CAMARGO, U.A.; NACHTIGAL, J.C.; MAIA, J.D.G; OLIVEIRA, P.R.D.; PROTAS, J.F.S. BRS Morena: nova cultivar de uva preta de mesa sem semente. Bento Gonçalves: Embrapa Uva e Vinho, 2003. 4p. (Comunicado Técnico, 46).

CANTWELL, M. Postharvest handling systems: minimally processed fruits and vegetables. In: KADER, A.A. (Ed.). Postharvest technology of horticultural crops. Oakland: University of California, 1992.p. 277-281.

CANTWELL, M. Preparation and quality of fresh- 
produce. In: ENCONTRO NACIONAL SOBRE PROCESSAMENTO MÍNIMO DE FRUTAS E HORTALIÇAS, 2., 2000, Viçosa. Palestras. Viçosa: UFV, 2000. p.150-172.

CHITARRA, M.I.F. Processamento mínimo de frutos e hortaliças. Viçosa: Centro de Produções Técnicas, 1998. 88p.

CHITARRA, M.I. Alterações bioquímicas do tecido vegetal com o processamento mínimo. In: SEMINÁRIO SOBRE HORTALIÇAS MINIMAMENTE PROCESSADAS, 1999, Piracicaba. Palestra... Piracicaba:ESALQ-USP, 1999. 9p. Apostila.

CHITARRA, A.B.; ALVES, R.E. Tecnologia de póscolheita para frutas tropicais. Fortaleza: Instituto Frutal/Sindifruta, 2001. v.1, 314p.

CHITARRA, M.I.F.; CHITARRA, A.B. Pós-colheita de frutos e hortaliças: fisiologia e manuseio. Lavras: ESAL-FAEPE, 2005, 785p.

COOMBE, B.G. Research on development and ripening of the grape berry. American Journal of Enology and Viticulture, Davis, v.43, n.1, p.101-110, 1992.

DETONI, A.M.; CLEMENTE, E.; BRAGA, G.C.; HERZOG, N.F. Uva 'Niagara Rosada' cultivada no sistema orgânico e armazenada em diferentes temperaturas. Ciência e Tecnologia de Alimentos, Campinas, v.25, n.3, p.546-552, 2005.

DURIGAN, J.F.; SARGENT, E.A. Uso de melão Cantaloupe na produção de produtos minimamente processados. Alimentos e Nutrição, São Paulo, v.10, p.69-77, 1999.

GRANGEIRO, L. C.; SOARES, J. M.; REIS, C. dos S.; DIGALVANINI, F; SILVA,A. V.C. da. Armazenamento refrigerado de uva, cultivar Superior Seedless produzida no Vale do São Francisco. In: CONGRESSO BRASILEIRO DE FRUTICULTURA, 17., 2002. Anais... Belém: SBF, 2002.p. 431.

KADER, A.A. (Ed.). Postharvest tecnology of horticultural crops. $2^{\text {nd }}$ ed. Oakland: University of California, 1992.296p.

KLUGE, R.A.; NACHTIGAL, J.C.; FACHINELLO, J.C.; BILHALVA, A.B. Fisiologia e manejo póscolheita de frutas de clima temperado. 2.ed. Pelotas: UFPel, 2002. 216p.

LEÃO, P.C. de S.; PEREIRA, F.M. Avaliação de seis variedades de uvas sem sementes no Submédio São Francisco. Pesquisa Agropecuária Brasileira,
Brasília, v.36, n.4, p.607-613, 2001.

LICHTER, A.; ZUTKHY, Y.; SONEGO, L.; DVIR, O.; KAPLUNOV, T.; SARIG, P.; BEN-ARIE, R. Ethanol controls postharvest decay of table grapes. Postharvest Biology and Technology, Amsterdan, v. 24, p. 301-308, 2002.

LIMA, M. A. C. de; ALVES, R. E.; ASSIS, J. S. de; FILGUEIRAS, H. A. C.; COSTA, J. T. A. Qualidade, fenóis e enzimas oxidativas de uva 'Itália' sob influência do cálcio, durante a maturação. Pesquisa Agropecuária Brasileira, Brasília, v.35, n.12, p.24932499, 2000.

MATTIUZ, B. H.; MIGUEL, A. C. A.; NACHTIGAL, J. C.; DURIGAN, J. F.; CAMARGO, U. A. Processamento mínimo de uvas de mesa sem semente. Revista Brasileira de Fruticultura, Jaboticabal, v.26, n.2, p.226-229, 2004.

MINOLTA CORP. Precise color communication: color control from feeling to instrumentation. Ramsey: Minolta Corporation Instrument Systems Division, 1994. 49p.

PINTO, S.A.A. Processamento mínimo de melão tipo Orange Flesh e de melancia 'Crimson Sweet'. 2002. 120 f. Dissertação (Mestrado em Produção Vegetal) - Faculdade de Ciências Agrárias e Veterinárias, Universidade Estadual Paulista, Jaboticabal, 2002.

RUFFNER, H. P.; BREM, S.; MALIPIERO, U. The physiology of acid metabolism in grape berry ripening. Acta Horticulturae, Wageningen, v.139, p.123-128, 1983.

SARZI, B. Conservação de abacaxi e mamão minimamente processados: associação entre o preparo, a embalagem e a temperatura de armazenamento. 2002. 100 f. Dissertação (Mestrado em Produção Vegetal) - Faculdade de Ciências Agrárias e Veterinárias, Universidade Estadual Paulista, Jaboticabal, 2002.

SAS INSTITUTE INC. SAS System for Microsoft Windows: release 9.1.3. Cary, 2004.

THÉ, P.M.P.; CARVALHO, V.D. de; ABREU, C.M.P. de; NUNES, R.P. de; PINTO, N.A.V.D. Efeito da temperatura de armazenamento e do estádio de maturação sobre a composição química do abacaxi cv. Smooth Cayenne L. Ciência e Agrotecnologia, Lavras, n.25, v.2, p.356-363, 2001.

WILEY, R.C. Frutas y hortalizas mínimamente procesadas y refrigeradas. Zaragoza: Acribia, 1997. 362 . 